\title{
Photoluminescence and Photoconductivity Dynamics in Semi-Insulating Epitaxial GaN Layers
}

\author{
E. Gaubas*, S. Jurš́̇nas, K. Kazlauskas, S. Miasojedovas, \\ J. VAITKus AND A. ŽUKAUSKas
}

Institute of Materials Science and Applied Research, Vilnius University

Sauletekio al. 10, 10223 Vilnius, Lithuania

\begin{abstract}
The transients of fast free-carrier recombination and of multi-trapping processes due to different species of defects have been investigated by photoluminescence and by contact and microwave photoconductivity. Three distinct stages of relaxation, namely, of stimulated emission, of recombination due to point defects and capture into trapping centers associated with dislocations, and a non-exponential stage with a stretched-exponent asymptotic decay ascribed to dislocations mediated multi-trapping were distinguished by correlated examination of time-resolved photoluminescence and photoconductivity transients.
\end{abstract}

PACS numbers: $61.72 . \mathrm{Hh}, 72.40 .+\mathrm{w}$

\section{Introduction}

GaN-based materials are widely used in fabrication of efficient short-wavelength light emitters $[1,2]$. The key issue of materials quality in GaN is high dislocation density $\left(10^{8}-10^{10} \mathrm{~cm}^{-2}\right)$, when radiative and non-radiative recombination depends on a dislocation type [3]. Luminescence and photoconductivity transients $[1,4]$ provide direct information on the carrier lifetime, which indicates the dominant recombination processes and defects.

In this paper, correlated investigations of the transients of time-resolved photoluminescence (TRPL), contact photoconductivity (CPC) and microwave absorption (MWA) are presented and analyzed in order to identify the behavior of different defects residing in the GaN epilayers. Distinct stages of relaxation, due to

*corresponding author; e-mail: eugenijus.gaubas@ff.vu.lt 
redistribution of the free-carrier capture flows between the centers of non-radiative recombination and multi-trapping, were clarified.

\section{Samples and experimental techniques}

A set of samples under investigation consisted of $2 \mu \mathrm{m}$ thick films of semi-insulating epitaxial GaN layer, grown by MOCVD on sapphire substrate with unintentionally doped $n$-GaN buffer. Room temperature PL measurements were carried out under excitation by continuous wave (cw) $\mathrm{He}-\mathrm{Cd}$ laser operating at $325 \mathrm{~nm}$ and pulsed (YAG: $\mathrm{Nd}^{3+}$ ) laser (266 nm wavelength). A toluene optical Kerr shutter with the temporal resolution of 20 ps was employed to display luminescence transients in the TRPL measurements. Measurements of the carrier decays were performed using contact photoconductivity and non-invasive microwave absorption techniques [4]. Pulsed excitation of the excess carriers was performed by a focused beam of either the 3rd harmonic $(355 \mathrm{~nm})$ or the fourth harmonic $(266 \mathrm{~nm})$ of YAG: $\mathrm{Nd}^{3+}$ lasers with either 10 ns or 20-30 ps pulses, in these time resolved experiments.

\section{Photoluminescence and photoconductivity dynamics}

In Fig. 1a, PL spectra under steady-state excitation (curves 1-4) and time integrated spectra under pulsed excitation (curves 5 and 6) are compared. At a relatively low excitation level, a spectrum consisting of three PL bands is observed.
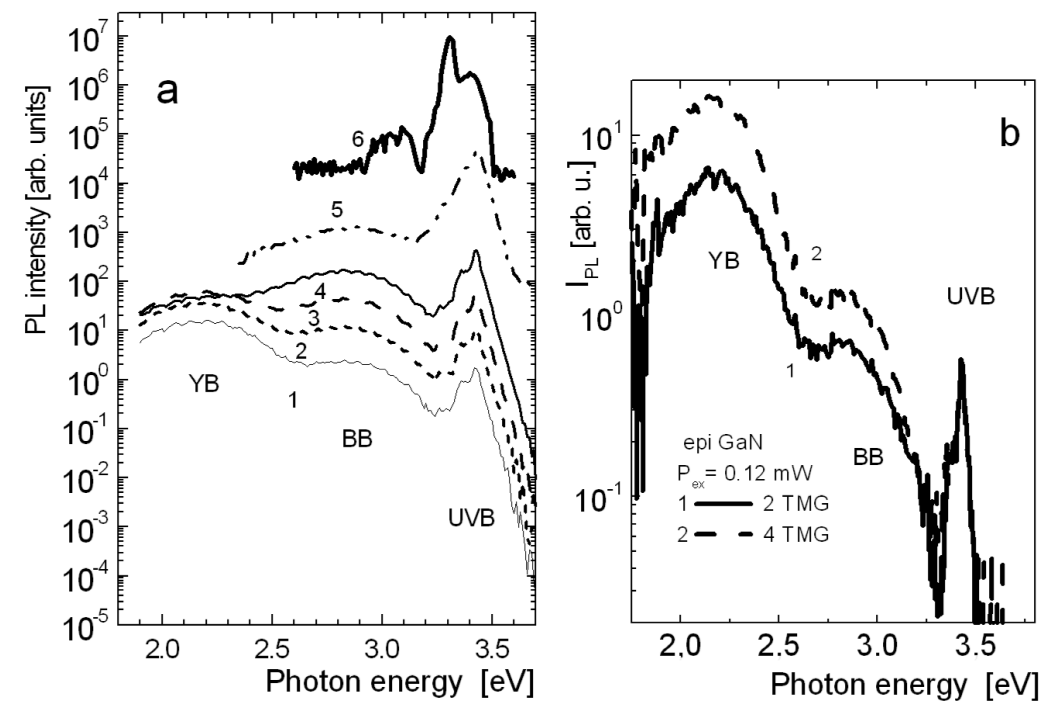

Fig. 1. PL spectra variations dependent on excitation density (a) and defect concentration (b) at fixed excitation density. Parameter increases with the curve number. 
The spectrum contains an ultraviolet (UV) band peaked at $3.42 \mathrm{eV}$ and attributed to band-to-band recombination. The UV band is accompanied with a blue (B) band with the peak in the range of $2.82-2.87 \mathrm{eV}$ and a yellow (Y) one peaked at $2.19 \mathrm{eV}$. Such a structure of the PL spectrum comprising the B band is inherent for $\mathrm{GaN}$ with high density of dislocations, due to the $60^{\circ}$ dislocations (A-type basal plane) that form a wide band of half-filled acceptor states [3]. Screw dislocations are known to be responsible for non-radiative recombination $[3,5]$. The observed $\mathrm{Y}$ band is ascribed to the complexes of Ga vacancy [3]. At high excitation levels the PL spectrum transforms remarkably (curves 5 and 6 in Fig. 1a). The Y-band PL disappears because of saturation of traps and nonlinear redistribution of the carrier capture flows with an increase in excess carrier density [6]. The UV band exhibits intense constituents of both stimulated and spontaneous emission, while an increase in intensity of the B-band PL band is smaller when compared with the UV band. Changes of the efficiency of the PL of Y-and B-bands were also observed due to variation of concentration of the grown-in defects at different trymethylgallium (TMG) flows applied (Fig. 1b). The B-band peak intensity increases linearly with excitation density, implying that the dislocation-related defects are capable of participating in recombination even at high excitation levels. However, the intensity of the $\mathrm{Y}$ band has a non-monotonic dependence on the excitation level with a remarkable decrease for excess carrier concentrations above $10^{15} \mathrm{~cm}^{-3}$ (compare curves 3 and 4 in Fig. 1a). This yields the trap concentration $N_{\mathrm{Y}}<10^{15} \mathrm{~cm}^{-3}$. Similarly, the absence of saturation of the "blue" levels implies that $N_{\mathrm{B}} \geq 10^{18} \mathrm{~cm}^{-3}$.
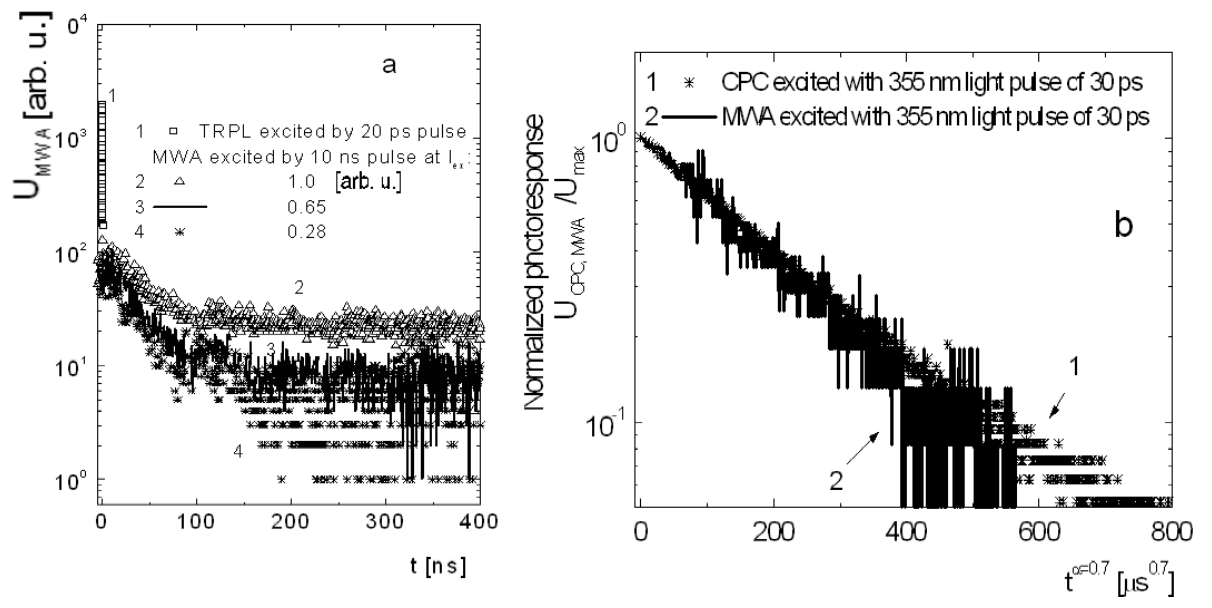

Fig. 2. (a) The transient of TRPL (1), measured at $3.4 \mathrm{eV}$ PL excited with $266 \mathrm{~nm}$ light 20 ps pulse, and MWA decays (2-4), recorded under $350 \mathrm{~nm}$ excitation with $10 \mathrm{~ns}$ pulses of different intensities. (b) Asymptotic CPC and MWA decays within layout of the stretched-exponent with the factor $\alpha=0.7$. 
The luminescence transient (Fig. 2a by curve 1), obtained by TRPL at the photon energy of $3.4 \mathrm{eV}$, contains an initial stage of PL relaxation due to stimulated emission, which is capable to rapidly reduce the carrier density to a value below the stimulation threshold, evaluated by carrier density of $n_{\mathrm{th}}=5 \times 10^{18} \mathrm{~cm}^{-3}[7]$. The subsequent luminescence decay is mostly determined by the rate of carrier capture into almost saturated defect levels and yields the carrier capture time of about 900 ps. The excess-carrier decays, revealed in the MWA and CPC transients, Fig. 2a, curves $2-4$, contain an initial stage with a fast component of a few nanoseconds dependent on the excitation density. The initial instantaneous lifetimes of the MWA decays are close to that estimated from the PL decay rate (curve 1 in Fig. 2a). The decay amplitude saturation effect (e.g. curve 2 in Fig. 2a) was found to be dependent on defect structure and concentration. Such a saturation phenomenon due to carrier multi-trapping is possible when the capture cross-section decreases with trap filling $[4,7]$. The initial trapping revealed in MWA and CPC transients can be explained by rapid hole trapping into dislocation-attributed defects, since in the $n$-type material no potential barrier for holes exists [8]. Then, the capture rate is determined solely by lateral diffusion towards dislocations [8]. Since the capture cross-section of these traps depends on their occupation and varies during relaxation of the excess carriers, the distribution of the trap depth in a cluster of dislocations is random, with a wide spectrum of traps [9]. The asymptotic nonexponential decay (which can be linearized in a logarithm of carrier density vs. $t^{\alpha}$ scale - Fig. 2b) component is also sensitive to defect density, as was established by comparison of the decays in samples of GaN layers fabricated using different TMG flows, in correspondence with PL data (Fig. 1b). This cluster determines the multi-trapping processes which decrease the recombination rate. The multitrapping transient behavior tends to stretched-exponent decay (Fig. 2b), when $I_{\mathrm{CPC}}(t)$ fits a stretched exponent $\exp \left[-(t / \tau)^{\alpha}\right]$ function [10]. The fraction dimension $d_{\mathrm{s}}[11]$ value of the disordered structure, evaluated as $d_{\mathrm{s}}=2 \alpha /(1-\alpha)$, is found to be $d_{\mathrm{s}}=4.7$. This $d_{\mathrm{s}}$ value implies that carriers motion is realized by percolation on an infinite cluster of dislocations net $\left(d_{\mathrm{s}}>2\right)[11]$ in the dislocations-rich GaN material.

\section{Conclusions}

The temporal evolution of the excess carrier decay was revealed on the time scale ranging from picoseconds to milliseconds and exhibited three distinct stages, namely, a stage of stimulated emission followed by exponential decay due to non-radiative centers, an intermediate stage due to dynamic screening of dislocation-related trapping centers, and an asymptotic stretched-exponent decay ascribed to disorder. In the asymptotic range, the excess carrier decay fits well the multitrapping model described by stretched-exponent approximation with the time-stretching factor $\alpha=0.7$, due to an infinite cluster of dislocations net. 


\section{Acknowledgments}

Prof. A. Sakai, Tokushima University, is acknowledged for provided GaN samples. This work was performed in the frame of the CERN RD50 Project and was partially supported by Lithuanian State Science and Studies Foundation.

\section{References}

[1] S. Nakamura, G. Fasol, The Blue Laser Diode: GaN Based Light Emitters and Lasers, Springer-Verlag, Berlin 1997.

[2] A. Žukauskas, M.S. Shur, R. Gaska, Introduction to Solid-State Lighting, Wiley, New York 2002.

[3] M. Albrecht, H.P. Strunk, J.L. Weyher, I. Grzegory, S. Porowski, T. Wosinski, J. Appl. Phys. 92, 2000 (2002).

[4] E. Gaubas, Lithuanian J. Phys. 43, 145 (2003).

[5] J. Bai, T. Wang, Y. Izumi, S. Sakai, J. Cryst. Growth 223, 61 (2001).

[6] S. Juršėnas, S. Miasojedovas, G. Kurilčik, A. Žukauskas, P.R. Hageman, Appl. Phys. Lett. 83, 66 (2003).

[7] P. Landsberg, Recombination in Semiconductors, Cambridge University Press, Cambridge 1991.

[8] H.F. Mataré, Defect Electronics in Semiconductors, Wiley-Interscience, New York 1971.

[9] G. Pfister, H. Sher, Adv. Phys. 27, 747 (1978).

[10] L. Pavesi, J. Appl. Phys. 80, 216 (1996).

[11] S. Havlin, D. Ben-Avraham, Adv. Phys. 51, 187 (2002). 